Artigo / Article

\title{
O transplante autólogo de células-tronco hematopoéticas no tratamento do Mieloma Múltiplo
}

Autologous hematopoietic stem cell transplant for Multiple Myeloma

Angelo Maiolino ${ }^{1}$

Roberto J. P. Magalhães ${ }^{2}$

\begin{abstract}
A quimioterapia em altas doses seguida de transplante autólogo de células-tronco hematopoéticas vem se constituindo ao longo das últimas décadas em um importante instrumento terapêutico, devendo fazer parte da estratégia de tratamento da maior parte dos pacientes com mieloma múltiplo, particularmente daqueles com idade inferior a 65 anos. Pelo menos dois importantes estudos randomizados mostraram vantagens para esta estratégia quando comparadas à quimioterapia convencional. No entanto, a quase totalidade destes pacientes irá recair, necessitando de algum tratamento adicional. A utilização de um segundo transplante, manutenção com talidomida e a introdução de novas drogas como o bortezomibe poderão representar um avanço, melhorando os resultados da estratégia de tratamento do mieloma múltiplo. Rev. bras. hematol. hemoter. 2007;29(1):36-41.
\end{abstract}

Palavras-chave: Mieloma múltiplo; transplante de células-tronco hematopoéticas; autólogo.

\section{Quimioterapia em altas doses sem suporte com células hematopoéticas}

A quimioterapia em altas doses no mieloma múltiplo (MM) foi introduzida no início dos anos 80 pelo grupo do hospital Royal Marsden (Reino Unido). Sessenta e três pacientes com MM recém-diagnosticados participaram de um estudo de fase II entre novembro de 1981 e abril de 1986. Todos os pacientes receberam melfalano em altas doses $\left(140 \mathrm{mg} / \mathrm{m}^{2}\right)$ sem suporte de células hematopoéticas. A taxa global de resposta foi de $82 \%$, com remissão completa (RC) de $32 \%$, bem maior que os $5 \%$ obtidos pela quimioterapia convencional (QT). ${ }^{1} \mathrm{O}$ principal inconveniente foi a alta mortalidade (16\%) relacionada ao tratamento (MRT). A sobrevida global (SG) mediana do grupo inteiro foi de quatro anos, e a sobrevida livre de evento (SLE) mediana foi de 16 meses.
Com a introdução do resgate hematopoético ocorreu uma considerável redução da toxicidade, e vários grupos em todo o mundo iniciaram estudos clínicos explorando esta prática.

\section{Estratégia da Universidade de Arkansas - Total Therapy I}

O grupo da Universidade de Arkansas (EUA) explorou uma abordagem alternativa para pacientes com MM recémdiagnosticado. O protocolo Total Therapy I incluiu uma fase de indução inicial com QT (VAD, três ou quatro ciclos), seguida de coleta de medula óssea ou células progenitoras de sangue periférico. Ciclofosfamida ( $\left.4 \mathrm{~g} / \mathrm{m}^{2}\right)$ e GM-CSF foram usados para a mobilização das células progenitoras. Após a coleta, um regime sem resistência cruzada (EDAP - etoposide, dexametasona, doxorrubicina e cisplatina) foi utilizado para

${ }^{1}$ Professor adjunto, Departamento de Clínica Médica, Serviço de Hematologia do Hospital Universitário Clementino Fraga Filho, Universidade Federal do Rio de Janeiro. Coordenador do Programa de Transplante de Medula Óssea do Hospital Universitário Clementino Fraga Filho

${ }^{2}$ Médico do Serviço de Hematologia do Hospital Universitário Clementino Fraga Filho, Universidade Federal do Rio de Janeiro.

Correspondência: Angelo Maiolino

Hospital Universitário Clementino Fraga Filho

Av. Brigadeiro Trompowsy, s/no - Cidade Universitária

21941-590 - Ilha do Fundão - Rio de Janeiro-RJ - Brasil

Tel: (21)2562-2462

E-mail: maiolino@hucff.ufrj.br 
aumentar a citorredução antes do Auto TMO. Um primeiro transplante foi realizado após condicionamento com melfalano $200 \mathrm{mg} / \mathrm{m}^{2}$ (MEL 200), seguido de um segundo, semelhante, três a seis meses depois. Os pacientes que não alcançaram pelo menos resposta parcial com o primeiro transplante receberam um regime de condicionamento diferente do inicial, com MEL 140 e Irradiação Corporal Total (ICT). Depois dos transplantes, os pacientes receberam IFN-2 $\alpha$ até que ocorresse a recidiva ou progressão da doença. Foram avaliados 231 pacientes com idade mediana de 51 anos (50 pacientes com idade $\geq 60$ anos), dos quais $9 \%$ tinham creatinina acima de $2,0 \mathrm{mg} / \mathrm{dl}$. Noventa por cento dos pacientes completaram a fase de indução, 84\% receberam um tratamento com altas doses, e 71\% receberam dois Auto TMO. O aumento progressivo das taxas de resposta após cada fase do protocolo foi evidente. A taxa de RC foi de 5\% após o VAD, 30\% após o primeiro transplante e $41 \%$ após o segundo transplante. Uma análise por intenção de tratamento de todos os 231 pacientes revelou uma SLE mediana em cinco anos de $42 \%$, e SG de $58 \%$. A mortalidade relacionada ao transplante foi de $1 \%$ após o primeiro e $4 \%$ após o segundo transplante. Os fatores associados a melhor prognóstico foram a ausência de anormalidades citogenéticas desfavoráveis (11q-e del13) e baixos níveis de $\beta_{2}$-microglobulina. ${ }^{2}$ Os resultados do protocolo foram comparados em um estudo caso-controle com os de pacientes tratados com QT, pelo Southwest Oncology Group (SWOG). Os pacientes foram pareados por idade, nível de $\beta_{2}$-microglobulina e creatinina. O Total Therapy I foi superior em termos de resposta parcial (85\% contra 52\%, $p=0,0001$ ), SLE (49 contra 20 meses, $\mathrm{p}=0,0001)$ e SG (62 contra 46 meses, $\mathrm{p}=0,003){ }^{3}$

\section{O primeiro estudo randomizado: IFM 90}

O Intergroupe Francophone du Myélome (IFM) conduziu um estudo randomizado comparando QT com quimioterapia em altas doses seguida de transplante de células hematopoéticas (Auto TMO) para pacientes recém-diagnosticados com MM em estágio II ou III. Os pacientes foram randomizados no momento do diagnóstico. O regime de QT foi o VMCP (vincristina, melfalano, ciclofosfamida e prednisona) / VBAP (vincristina, carmustina, doxorrubicina e prednisona). Os pacientes no braço de QT receberam um total de 12 ciclos, e o grupo de Auto TMO recebeu de quatro a seis ciclos do mesmo tratamento como indução, seguido de coleta de medula óssea autóloga. Os pacientes receberam Auto TMO com melfalano $140 \mathrm{mg} / \mathrm{m}^{2}$ (MEL 140) e ICT com 8 Gy. Ambos os grupos receberam interferon- $\alpha$ (IFN- $\alpha$ ) como manutenção até a recidiva ou progressão da doença. No total, 200 pacientes foram avaliados. Os pacientes em ambos os grupos eram comparados com relação à idade, sexo, estádio, isotipo e nível de $\beta_{2}$-microglobulina. A RC foi definida como ausência da paraproteína na eletroforese do soro e da urina, e menos de $5 \%$ de plasmócitos no mielograma. Resposta parcial muito boa (RPMB) foi definida como redução de $90 \%$ no nível de paraproteína. O Auto TMO melhorou significativamente as taxas de resposta em comparação com a QT: 38\% dos pacientes no braço Auto TMO alcançaram RC ou RPMB, contra $14 \%$ dos pacientes no grupo de QT ( $p<0,001)$. A SLE e a SG também melhoraram com o Auto TMO, chegando a medianas de 28 e 57 meses, contra 18 e 44 meses no grupo da QT. A SLE e a SG em sete anos foram $8 \%$ e $25 \%$ no braço de QT, contra $16 \%$ e $43 \%$ no braço de Auto TMO ( $p=0,01$ e p $=0,03$, respectivamente). Apesar de o estudo IFM 90 ter mostrado melhora significativa em termos de taxa de resposta, SLE e SG para os pacientes tratados com Auto TMO, a SLE em sete anos foi de apenas $16 \%$, sem que se observasse um plateau da curva no braço de Auto TMO, sugerindo que novas estratégias terapêuticas deveriam ser introduzidas e

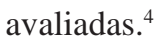

Pelo menos mais cinco estudos randomizados foram publicados na seqüência ao IFM, a maioria deles mostrando vantagem para o Auto TMO (Tabela 1).

Tabela 1. Comparação entre os principais estudos randomizados de quimioterapia convencional (QT) e quimioterapia em altas doses com resgate hematopoético (Auto TMO) no tratamento do Mieloma

\begin{tabular}{|c|c|c|c|c|c|c|}
\hline & & $\begin{array}{c}\text { Pacientes } \\
\mathrm{N}\end{array}$ & $\begin{array}{c}\text { Idade } \\
\text { anos }\end{array}$ & $\mathrm{CR}$ & $\begin{array}{c}\text { EFS } \\
\text { meses }\end{array}$ & $\begin{array}{c}\text { OS } \\
\text { meses }\end{array}$ \\
\hline Attal et $a l^{4}$ & $\begin{array}{c}\text { QT } \\
\text { Auto MO }\end{array}$ & $\begin{array}{l}100 \\
100\end{array}$ & $<65$ & $\begin{array}{c}5 \% \\
22 \%\end{array}$ & $\begin{array}{l}18 \\
27\end{array}$ & $\begin{array}{l}44 \\
57\end{array}$ \\
\hline Fermand et $a l^{7}$ & $\begin{array}{c}\text { QT } \\
\text { Auto TMO }\end{array}$ & $\begin{array}{l}96 \\
94\end{array}$ & $55-65$ & $\begin{array}{l}- \\
-\end{array}$ & $\begin{array}{l}19 \\
24\end{array}$ & $\begin{array}{l}50,4 \\
55,3\end{array}$ \\
\hline Barlogie et $a^{21}$ & $\begin{array}{c}\text { QT } \\
\text { Auto TMO }\end{array}$ & $\begin{array}{l}255 \\
261\end{array}$ & $<70$ & - & $\begin{array}{l}16 \\
17\end{array}$ & $\begin{array}{l}42 \\
37\end{array}$ \\
\hline Child et $a^{22}$ & $\begin{array}{c}\text { QT } \\
\text { Auto TMO }\end{array}$ & $\begin{array}{l}200 \\
201\end{array}$ & $<64$ & $\begin{array}{c}9 \% \\
44 \%\end{array}$ & $\begin{array}{l}20 \\
32\end{array}$ & $\begin{array}{l}42,3 \\
54,8\end{array}$ \\
\hline Blade et $a^{23}$ & $\begin{array}{c}\text { QT } \\
\text { Auto TMO }\end{array}$ & $\begin{array}{l}83 \\
81\end{array}$ & $<65$ & $\begin{array}{l}11 \% \\
30 \%\end{array}$ & $\begin{array}{l}34 \\
42\end{array}$ & $\begin{array}{l}56 \\
61\end{array}$ \\
\hline Palumbo et $a^{15}$ & $\begin{array}{c}\text { QT } \\
\text { Auto TMO }\end{array}$ & $\begin{array}{l}98 \\
97\end{array}$ & $50-70$ & $\begin{array}{c}7 \% \\
26 \%\end{array}$ & $\begin{array}{l}16 \\
28\end{array}$ & $\begin{array}{l}43 \\
58\end{array}$ \\
\hline
\end{tabular}

N: número; CR: remissão completa; EFS: sobrevida livre de eventos; OS: sobrevida global; QT: quimioterapia convencional; Auto TMO: transplante autólogo de medula óssea.

\section{Transplante único ou transplante duplo?}

A chave para a cura do MM pode estar na máxima intensificação possível do tratamento para a obtenção de uma remissão completa (RC). A RC afeta de forma positiva as taxas de SLE e SG. ${ }^{5}$ O grupo da Universidade de Arkansas considera o duplo transplante como a melhor forma de intensifi- 
cação do tratamento e utilizou esta estratégia como parte do protocolo Total Therapy I. O grupo francês (IFM) explorou esta estratégia em um estudo randomizado. No estudo IFM 94, 399 pacientes com MM recém-diagnosticado e idade inferior a 60 anos foram randomizados para receber um Auto TMO único, após MEL 140 e ICT (8 Gy), ou um Auto TMO duplo, utilizando MEL 140 no primeiro e MEL140 com ICT (8 Gy) no segundo. Os pacientes foram inicialmente tratados com três a quatro ciclos de VAD. As características e fatores de risco dos pacientes eram semelhantes entre os quatro grupos. As taxas de resposta (RC + RPMB) não foram significativamente diferentes entre os dois grupos (42\% no transplante único e $50 \%$ no duplo). No entanto, os pacientes submetidos ao duplo transplante apresentaram maior SLE em sete anos ( $20 \%$ contra $10 \%$; p < 0,03) e SG em sete anos ( $42 \%$ contra $21 \%$; $<<0,01)$. Este benefício, no entanto, ficou mais evidente apenas para os subgrupos de pacientes que foram maus respondedores ao VAD (> de 50\% de redução da proteína M) ou ao primeiro transplante (> de $90 \%$ de redução da proteína M) ${ }^{6}$

Mais quatro grupos compararam a estratégia único versus duplo transplante (Tabela 2).

Tabela 2. Comparação entre os principais estudos randomizados de transplante autólogo único versus duplo no tratamento do mieloma

\begin{tabular}{|c|c|c|c|c|c|c|}
\hline & & $\begin{array}{c}\text { Pacientes } \\
\mathrm{N}\end{array}$ & $\begin{array}{l}\text { Idade } \\
\text { anos }\end{array}$ & $\mathrm{CR}$ & $\begin{array}{c}\text { EFS } \\
\text { meses }\end{array}$ & $\begin{array}{c}\text { OS } \\
\text { meses }\end{array}$ \\
\hline Attal et al ${ }^{6}$ & $\begin{array}{l}\text { Único } \\
\text { Duplo }\end{array}$ & $\begin{array}{l}200 \\
202\end{array}$ & $<61$ & $\begin{array}{l}42 \% \\
50 \%\end{array}$ & $\begin{array}{l}25 \\
30\end{array}$ & $\begin{array}{l}44 \\
57\end{array}$ \\
\hline Fermand et al ${ }^{24}$ & $\begin{array}{l}\text { Único } \\
\text { Duplo }\end{array}$ & $\begin{array}{l}113 \\
114\end{array}$ & $<56$ & $\begin{array}{l}39 \% \\
37 \%\end{array}$ & $\begin{array}{l}31 \\
33\end{array}$ & $\begin{array}{l}50,4 \\
55,3\end{array}$ \\
\hline Cavo et al ${ }^{25}$ & $\begin{array}{l}\text { Único } \\
\text { Duplo }\end{array}$ & $\begin{array}{l}110 \\
110\end{array}$ & $<61$ & $\begin{array}{l}31 \% \\
43 \%\end{array}$ & $\begin{array}{l}21 \\
31\end{array}$ & $\begin{array}{l}53 \\
58\end{array}$ \\
\hline Segeren et al ${ }^{26}$ & $\begin{array}{l}\text { Único } \\
\text { Duplo }\end{array}$ & $\begin{array}{l}129 \\
132\end{array}$ & $<66$ & $\begin{array}{l}13 \% \\
29 \%\end{array}$ & $\begin{array}{l}21 \\
22\end{array}$ & $\begin{array}{l}50 \\
47\end{array}$ \\
\hline
\end{tabular}

N: número; CR: remissão completa; EFS: sobrevida livre de eventos; OS: sobrevida global.

Estes estudos mostraram resultados diferentes do grupo francês. Em nenhum deles ficou demonstrado vantagem em termos de sobrevida global para o grupo de pacientes submetidos ao duplo transplante, e apenas no estudo do grupo italiano ficou demonstrado vantagem em termos de SLE.

\section{Qual o melhor momento para a realização do transplante?}

A questão de qual seria o melhor momento no curso da doença (precoce ou tardio) para a realização de Auto TMO foi explorada em um único estudo randomizado que comparou ambas as estratégias. Neste estudo foram incluídos 185 pacientes, dos quais 91 receberam Auto TMO após indução rápida com QT (transplante precoce), e 94 receberam Auto
TMO somente após ocorrência de recidiva ou progressão da doença (transplante tardio). Os resultados foram semelhantes quanto à mortalidade relacionada ao transplante (MRT) e SG, mas os pacientes transplantados precocemente receberam menos ciclos de quimioterapia e permaneceram assintomáticos por mais tempo que os transplantados tardiamente.

\section{Regimes de condicionamento}

A grande maioria dos regimes de condicionamento utilizados no MM baseia-se na combinação de melfalano como agente único ou combinado a ICT, em doses diferentes $\left(140 \mathrm{mg} / \mathrm{m}^{2}\right.$ a $\left.220 \mathrm{mg} / \mathrm{m}^{2}\right)$. Combinações incluindo outros agentes alquilantes já foram utilizadas, mas nenhuma apresentou vantagens significativas quando comparada ao melfalano. ${ }^{8} \mathrm{O}$ grupo francês conduziu um estudo randomizado (IFM 95), comparando dois regimes diferentes de Auto TMO para MM recém-diagnosticado em 282 pacientes. No braço A, 140 pacientes foram tratados com MEL 140 e ICT 8 Gy, e no braço B, 142 pacientes foram tratados com MEL 200. A toxicidade foi significativamente menor no grupo tratado sem ICT (menor duração de neutropenia, de trombocitopenia e de internação; menos transfusões; menos mucosite grave). Cinco mortes por toxicidade ocorreram no braço com ICT, contra nenhuma no outro braço. A SLE mediana foi semelhante (21 contra 20,5 meses), mas a SG em 45 meses foi significativamente maior no grupo sem ICT $(65,8 \%$ contra $45,5 \%, p=0,05)$. Os autores concluíram que o MEL 200 é menos tóxico, reduz a MRT e melhora a SG. ${ }^{9}$

\section{Estratégias pós-transplante}

O tratamento com altas doses aumenta a taxa de resposta e prolonga a SLE e SG em pacientes com MM, mas, infelizmente, poucos pacientes se algum - são verdadeiramente curáveis. Portanto, o conceito de que o transplante é a modalidade terapêutica final, sem a necessidade de tratamento adicional, não se aplica ao MM como acontece em outras doenças. No MM, o transplante deve ser considerado apenas uma entre as várias etapas do tratamento; um conceito mais parecido com o do tratamento da leucemia linfóide aguda, por exemplo. A exploração de estratégias pós-transplante é, assim, muito importante para a melhora dos resultados.

As abordagens clássicas, utilizadas em regime de QT, é a manutenção com IFN- $\alpha$. Em um estudo, 85 pacientes foram randomizados para receber IFN- $\alpha\left(3 \times 10^{6} \mathrm{UI}\right.$ três vezes por semana) até a recidiva ou progressão, contra nenhum tratamento adicional. Após mediana de 52 meses, a sobrevida livre de progressão (SLP) no grupo tratado com IFN- $\alpha$ era de 
46 meses, contra 27 meses no grupo-controle $(p=0,0025)$. No entanto, a vantagem de sobrevida desapareceu em uma análise posterior, com seguimento mais longo. ${ }^{10}$

Outra estratégia de consolidação pós-transplante foi avaliada pelo grupo da Universidade de Arkansas. O regime de poliquimioterapia DCEP (dexametasona, ciclofosfamida, etoposide e cisplatina), que já havia sido utilizado com sucesso para o tratamento de recidivas pós-transplante, ${ }^{11}$ foi testado como consolidação pós-transplante (quatro ciclos). Setenta e um pacientes receberam DCEP e foram comparados a um grupo histórico de 142 pacientes, pareados pela presença de anormalidades cromossômicas desfavoráveis e níveis de $\beta_{2}$-microglobulina. Os pacientes que receberam DCEP apresentaram melhor SLE (37 contra 15 meses, $\mathrm{p}=0,002$ ) e SG (34 contra 13,5 meses, $p=0,004) .{ }^{12}$

A talidomida, isoladamente ou em combinação com a dexametasona, tem sido utilizada em diversos centros para o tratamento da recaída pós-transplante. Seu papel como tratamento de manutenção e como terapia de primeira linha também é alvo de grande interesse. Recentemente foram divulgados os resultados do estudo randomizado IFM 99-02, que comparou em um subgrupo de pacientes de baixo risco (ausência de deleção do cromossoma 13 e/ou beta 2-microglobulina $<3 \mathrm{mg} / \mathrm{dl}$ ) três estratégias diferentes de manutenção: ausência de manutenção, uso do pamidronato ou uso do pamidronato associado a talidomida. O tratamento de indução proposto foi com três ciclos de VAD e, na seqüência, duplo transplante com MEL 140 + MEL 200.

O grupo que utilizou talidomida com pamidronato obteve vantagens em termos de SLE e de SG quando comparado aos outros dois grupos, sendo a conclusão final do estudo que a adição da talidomida após o transplante aumenta a duração da resposta dos pacientes com MM de baixo risco. ${ }^{13}$

\section{Transplantes em pacientes idosos}

O MM é predominantemente diagnosticado em pacientes com idade avançada (acima de 65 anos), o que, na maioria das vezes, contra-indica a realização do transplante. O grupo da Universidade de Arkansas avaliou 159 pacientes com mais de 70 anos. Destes, 94 (59\%) foram submetidos a coleta de células-tronco. Em 70 pacientes foi possível obter uma coleta adequada. Estes pacientes foram então submetidos a Auto TMO. Os 25 primeiros, que receberam MEL 200, apresentaram alta mortalidade (16\%). Os 45 subseqüentes receberam MEL 140, e a MRT caiu para $2 \%$. Trinta e um pacientes ainda tiveram condições clínicas para receber o segundo transplante. Os autores concluíram ser factível o Auto TMO nesta população, devendo-se, no entanto, utilizar doses menores de quimioterapia (MEL 140). ${ }^{14}$

Palumbo explorou, em uma população maior que 65 anos, a utilização de uma dose intermediária que constava de dois cursos consecutivos de MEL 100 com resgate hematopoético comparando com o tratamento oral convencional melfalano - prednisona (MP) em um total de seis ciclos. Neste estudo, o esquema de dose intermediária demonstrou maiores taxas de resposta completa ( $25 \%$ contra $8 \%$ ), de SLE (31\% contra $18 \%$ $\mathrm{p}=0.01)$ e de $\mathrm{SG}(73 \%$ contra $58 \% \mathrm{p}=0.01)$ em três anos de seguimento. Houve maior incidência de mucosite e febre, sendo a maior causa de mortalidade a progressão de doença nos dois primeiros meses do diagnóstico. ${ }^{15}$ Este mesmo autor conjuntamente com o grupo Italiano GIMEMA publicou um estudo comparativo randomizado entre dois esquemas orais: o MP e o MP adicionado da talidomida (MP-TAL). Os resultados apontam para a superioridade do MP-TAL com maiores taxas de resposta ( $76 \%$ contra $47,6 \%$ ) e SLE em dois anos (54\% contra $27 \% \mathrm{p}=0.0006)$. Houve maior incidência global de eventos adversos grau 3-4 no MP-TAL (48\% x $25 \% \mathrm{p}=0.0002$ ), sendo os mais observados neuropatia periférica, infecção e tromboembolismo pulmonar. Os autores sugeriram a introdução de heparina de baixo peso molecular profilática para contornar este último problema relacionado a talidomida. ${ }^{16}$

Por fim foram divulgados os resultados parciais do esperado estudo IFM 99-06 que, em uma população de pacientes idosos (65-75 anos), comparou três estratégias diferentes: o esquema MP, MP-TAL ou dois ciclos de MEL $100 \mathrm{com}$ resgate hematopoético. O esquema MP-TAL foi superior ao tratamento convencional e ao MEL100, atingindo melhores resultados em termos de tempo de SG e SLP. Entretanto, quando o MP foi comparado ao MEL 100, não foi demonstrada diferença estatisticamente significativa. A conclusão dos autores foi que, até o atual momento, em pacientes idosos inelegíveis para o transplante, o tratamento de referência a ser oferecido é o MP-TAL. ${ }^{17}$

\section{Transplantes em pacientes com insuficiência renal}

Insuficiência renal pode ser diagnosticada em cerca de $20 \%$ dos pacientes com MM em algum momento da evolução da doença. ${ }^{18}$ Pelo menos um estudo demonstrou que a farmacocinética do melfalano é comparável entre pacientes com ou sem insuficiência renal. ${ }^{19} \mathrm{O}$ grupo da Universidade de Arkansas avaliou 81 pacientes com MM e insuficiência renal no momento do transplante. Destes, 38 encontravamse em programa de diálise. A mediana de idade era de 53 anos (29 - 69 anos). Para a mobilização de células-tronco, utilizouse G-CSF isoladamente em 51 pacientes, e quimioterapia mais G-CSF em 27 pacientes. MEL 200 foi utilizado como quimioterapia em altas doses em 60 pacientes (27 em diálise). Devido à toxicidade excessiva, os 21 pacientes subseqüentes receberam dose reduzida de melfalano $\left(140 \mathrm{mg} / \mathrm{m}^{2}\right)$. Um segundo transplante foi efetuado em 31 pacientes (11 em diálise). A MRT foi de $6 \%$ após o primeiro e $13 \%$ após o segundo Auto TMO. Os autores concluíram que a insuficiência renal não tem impacto na qualidade da coleta de células-tronco e não prolonga o tempo de recuperação do enxerto ("pega"). 
O regime MEL 140 tem toxicidade aceitável, com eficácia comparável à do MEL 200, e deve ser o regime de escolha neste grupo de pacientes. ${ }^{20}$

\section{Conclusão}

O Auto TMO vem se consolidando nos últimos anos como um instrumento de fundamental importância, devendo quase sempre fazer parte da estratégia terapêutica no MM.

Um melhor conhecimento da biologia e da fisiopatologia da doença, aliado a um maior conhecimento dos fatores de risco relacionados à resposta terapêutica, permitirão prolongar consideravelmente a sobrevida, melhorar a qualidade de vida e, eventualmente, curar uma fração significativa de pacientes.

\section{Recomendações}

- Tratamento de primeira linha para pacientes com idade inferior a 65 anos com bom PS: Transplante autólogo com células-tronco hematopoéticas do sangue periférico.

- $\quad$ Melhor regime de condicionamento: MEL 200 mg/m².

- $\quad$ Melhor momento do Auto TMO: inicial após indução.

- Duplo transplante: pacientes que não atingiram resposta parcial muito boa após o $\mathrm{D}+100$ do primeiro Auto TMO.

- Mobilização: objetivo - coletar dose celular esti-

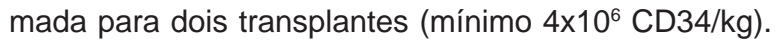

- $\quad$ Transplante idoso: pacientes maiores de 65 anos com bom PS podem ser considerados para Auto TMO. No entanto, evidências apontam para uma equivalência de resultados quando comparado à quimioterapia convencional.

- $\quad$ Transplante em insuficiência renal: Reduzir condicionamento para MEL $140 \mathrm{mg} / \mathrm{m}^{2}$.

\footnotetext{
Abstract

High dose chemotherapy followed by autologous stem cell transplantation has been recognized as an important step in the treatment of multiple myeloma. At least two well designed randomized studies showed better outcomes in patients treated with high doses compared to those treated with conventional chemotherapy. Nowadays, autologous stem cell transplantation should be considered for all under 65-year-old patients. Although autologous stem cell transplantation has modified the prognosis of myeloma, almost all patients still relapse some time after a single transplant, and then another therapeutic approach becomes necessary. With the aim of improving the results in the treatment of
}

myeloma, new approaches including tandem stem cell transplantation, maintenance with thalidomide and new drugs such as bortezomib are being tested. Strategies including these approaches and autologous stem cell transplantation may improve the results of the treatment of myeloma in the future. Rev. bras. hematol. hemoter. 2007;29(1):36-41.

Key words: Multiple myeloma; hematopoietic stem cell transplantation; autologous.

\section{Referências Bibliográficas}

1. Selby PJ, McElwain TJ, Nandi AC, Perren TJ, Powles RL, Tillyer CR, et al. Multiple myeloma treated with high dose intravenous melphalan. Br J Haematol 1987;66(1):55-62.

2. Barlogie B, Jagannath S, Desikan KR, Mattox S, Vesole D, Siegel $\mathrm{D}$, et al. Total therapy with tandem transplants for newly diagnosed multiple myeloma. Blood;1999;93(1):55-65.

3. Barlogie B, Jagannath S, Vesole DH, Naucke S, Cheson B, Mattox $\mathrm{S}$, et al. Superiority of tandem autologous transplantation over standard therapy for previously untreated multiple myeloma. Blood;1997;89(3):789-93.

4. Attal M, Harousseau JL, Stoppa AM, Sotto JJ, Fuzibet JG, Rossi JF, et al. A prospective, randomized trial of autologous bone marrow transplantation and chemotherapy in multiple myeloma. Intergroupe Francais du Myelome. N Engl J Med 1996;335(2):91-7.

5. Harousseau JL, Attal M, Divine M, Marit G, Leblond V, Stoppa AM, et al. Autologous stem cell transplantation after first remission induction treatment in multiple myeloma: a report of the French Registry on autologous transplantation in multiple myeloma. Blood 1995;85(11):3.077-85.

6. Attal M, Harousseau JL, Facon T, Guilhot F, Doyen C, Fuzibet JG, et al. Single versus double autologous stem-cell transplantation for multiple myeloma. N Engl J Med 2003;349(26):2.495-502.

7. Fermand JP, Ravaud P, Chevret S, Divine M, Leblond V, Belanger $\mathrm{C}$, et al. High-dose therapy and autologous peripheral blood stem cell transplantation in multiple myeloma: up-front or rescue treatment? Results of a multicenter sequential randomized clinical trial. Blood 1998;92(9):3.131-6.

8. Dimopoulos MA, Alexanian R, Przepiorka D, Hester J, Andersson B, Giralt S, et al. Thiotepa, busulfan, and cyclophosphamide: a new preparative regimen for autologous marrow or blood stem cell transplantation in high-risk multiple myeloma. Blood 1993; 82(8):2.324-8

9. Moreau P, Facon T, Attal M, Hulin C, Michallet M, Maloisel F, et al. Comparison of $200 \mathrm{mg} / \mathrm{m}^{2}$ melphalan and 8 Gy total body irradiation plus $140 \mathrm{mg} / \mathrm{m}^{2}$ melphalan as conditioning regimens for peripheral blood stem cell transplantation in patients with newly diagnosed multiple myeloma: final analysis of the Intergroupe Francophone du Myelome 9502 randomized trial. Blood 2002; 99(3):731-5.

10.Cunningham D, Powles R, Malpas J, Raje N, Milan S, Viner C, et al. A randomized trial of maintenance interferon following high-dose chemotherapy in multiple myeloma: long-term follow-up results. Br J Haematol 1998;102(2):495-502.

11. Munshi N, Desikan KR, Jagannath S, Siegel D, Bracy D, Tricot G, et al. Dexamethasone, Cyclophosphamide, Etoposide and Cisplatinum (DCEP), an effective regimen for relapse after highdose chemotherapy and autologous transplant (AT). Blood 88 (suppl),586a.1996. Abstract

12. Fassas A, Tricot G. Results of high-dose treatment with autologous stem cell support in patients with multiple myeloma. Semin Hematol 2001;38(3):231-42. 
13. Attal M, Harousseau JL, Leyvraz S, Doyen C, Hulin C, Benboubker $\mathrm{L}$, et al. Maintenance therapy with thalidomide improves survival in multiple myeloma patients. Blood 2006;108:3.289-3.294.

14. Badros A, Barlogie B, Siegel E, Morris C, Desikan R, Zangari M, et al. Autologous stem cell transplantation in elderly multiple myeloma patients over the age of 70 years. Br J Haematol 2001; 114(3):600-7.

15. Palumbo A, Bringhen S, Petrucci MT, Musto P, Rossini F, Nunzi $\mathrm{M}$, et al. Intermediate-dose melphalan improves survival of myeloma patients aged 50 to 70 : results of a randomized controlled trial. Blood 2004;104(10):3.052-7.

16. Palumbo A, Bringhen S, Caravita T, Merla E, Capparella V, Callea $\mathrm{V}$, et al. Oral melphalan and prednisone chemotherapy plus thalidomide compared with melphalan and prednisone alone in elderly patients with multiple myeloma: randomised controlled trial. Lancet 2006;367(9513):825-31.

17. Facon T, Mary J, Harrousseau J, Rondon P. Superiority of MP plus Thalidomide over MP or autologous stem cell transplant of newly diagnosed elderly patients with multiple myeloma (IFM 99-06). ASCO Annual Meeting Proceedings 24[18 S]. 2006. Abstract

18. Alexanian R, Barlogie B, Dixon D. Renal failure in multiple myeloma. Pathogenesis and prognostic implications. Arch Intern Med 1990;150(8):1.693-5.

19. Tricot G, Alberts DS, Johnson C, Roe DJ, Dorr RT, Bracy D, et al. Safety of autotransplants with high-dose melphalan in renal failure: a pharmacokinetic and toxicity study. Clin Cancer Res 1996; 2(6): 947-52.

20. Badros A, Barlogie B, Siegel E, Roberts J, Langmaid C, Zangari M, et al. Results of autologous stem cell transplant in multiple myeloma patients with renal failure. Br J Haematol 2001;114 (4):822-9.

21. Barlogie B, Kyle RA, Anderson KC, Greipp PR, Lazarus HM, Hurd $\mathrm{DD}$, et al. Standard chemotherapy compared with high-dose chemoradiotherapy for multiple myeloma: final results of phase III US Intergroup Trial S9321. J Clin Oncol 2006;24(6): 929-36.

22. Child JA, Morgan GJ, Davies FE, Owen RG, Bell SE, Hawkins K, et al. High-dose chemotherapy with hematopoietic stem-cell rescue for multiple myeloma. N Engl J Med 2003; 348(19): 1.875-83.
23. Blade J, Rosinol L, Sureda A, Ribera JM, az-Mediavilla J, GarciaLarana J, et al. High-dose therapy intensification compared with continued standard chemotherapy in multiple myeloma patients responding to the initial chemotherapy: long-term results from a prospective randomized trial from the Spanish cooperative group PETHEMA. Blood 2005;106(12):3.755-9.

24. Fermand JP, Marallian J, Albert C. Single versus tandem high dose therapy (HDT) supported with autologous blood stem cell transplantation using unsellected or CD-34 enriched ABSC: preliminary results of a two by two design randomized trial in 230 young patients with multiple myeloma. Blood 98, 815a. 2001. Abstract

25. Cavo M, Tosi P, Zagmagni E. The Bologna 96 clinical trial of single versus double PBSC transplantation for previously untreated MM: results of an interin analysis. Blood 100[17a]. 2002. Abstract

26. Segeren CM, Sonneveld P, van der HB, Vellenga E, Croockewit AJ, Verhoef GE, et al. Overall and event-free survival are not improved by the use of myeloablative therapy following intensified chemotherapy in previously untreated patients with multiple myeloma: a prospective randomized phase 3 study. Blood 2003 Mar 15;101(6):2.144-51.

O tema apresentado e o convite ao(s) autor(es) consta da pauta elaborada pelo co-editor.

Avaliação: Co-editor e um revisor externo.

Publicado após revisão e concordância do editor.

Conflito de interesse: não declarado.

Recebido: 25/11/2006

Aceito: 15/01/2007 